\title{
Non-invasive estimation of the intracranial pressure waveform from the central aortic pressure waveform
}

\section{Karen Brastad Evensen ${ }^{1,2}$, Per Kristian Eide ${ }^{2,3}$}

Monitoring of intracranial pressure (ICP) is done in neurosurgical departments and intensive care units worldwide to aid the physician's management of patients with severe brain injury or disease. Clinical ICP measurements include neurosurgical penetration of the skull and insertion of a pressure sensor or drain into the brain parenchyma or the cerebral fluid spaces. This procedure entails risks of haemorrhage and infections, and requires expensive neurosurgical expertise. Currently, ICP monitoring is therefore restricted to critically ill individuals where the need to measure ICP outweighs the invasiveness of the procedure. A non-invasive and risk-free method for ICP monitoring would increase the clinical utility, and allow for ICP scoring in patient populations with less critical pathologies, where the role of ICP so far is unclear.

Given the potential benefits, numerous approaches have been explored in the search for successful non-invasive ICP estimation techniques. Examples include Imaging-Based Methods, Optic Nerve Sheath Diameter evaluation, Tympanic membrane displacement and Transcranial Doppler ultrasonography ${ }^{1,2}$. Another category is estimation of mean ICP or other ICP parameters from radial artery blood pressure measurements, sometimes in conjunction with additional measurements ${ }^{3-6}$. These are appealing approaches, as radial artery blood pressure is easily accessible and possible to monitor continuously. Our approach in the paper by Evensen et. al. ${ }^{7}$ falls into this category of non-invasive ICP estimation.

While most of the research presented in literature focuses on mean ICP, we took interest in the pulsatile part that is derived from cardiac activity. The pulsatile ICP is primarily driven by the beat-to-beat arterial blood pressure pulsations that pass through the intracranial compartment. As the ICP waveform is the cerebrospinal space's response to the excitations by the cardiac beat contractions, it has been suggested that the central aortic blood pressure waveform is a better source for non-invasive ICP estimation than the radial artery blood pressure waveform. A preliminary study reported that the central aortic blood pressure waveform were more similar to the ICP waveform than the radial artery blood waveform, which supported this proposal ${ }^{8}$. During the period of systole, the central aortic blood pressure waveform was reported to be almost identical to the ICP waveform and the augmentation index was similar to that of ICP. When comparing the pulsatile ICP and the radial artery blood pressure waveforms these were notably more different.

As the mean ICP level is the most commonly assessed parameter, the direct utility of the ICP waveform might not be evident. However, there are recent clinical studies that show that managing patients with intracranial bleeds (subarachnoid haemorrhage, SAH) according to

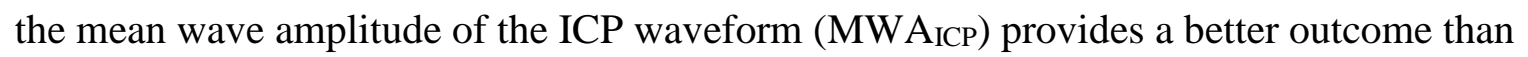
management according to the traditional mean ${ }^{4,9-12}$. In our study, we therefore focused on the MWA ${ }_{\text {ICP }}$ parameter, indicated to reflect the physical characteristics of the intracranial space, 
and aimed at estimating it non-invasively using estimates of the central aortic blood pressure waveforms. These were found from the radial artery blood pressure waveform using the SphygmoCor system (SphygmoCor®; AtCor Medical, West Ryde, NSW, Australia).

To evaluate the possible usefulness of the central aortic estimates for MWA $\mathrm{IIP}_{\mathrm{IC}}$ estimation, patient specific transfer functions were calculated using spectral analysis. The central aortic signals were used as input and the invasively obtained ICP signal as output. In total 29 patients were included in the study. All patients had idiopathic normal pressure hydrocephalus and ICP levels in the normal range. The maximum mean ICP of the cohort was $9.8 \mathrm{mmHg}$. In our study, we first calculated patient specific transfer functions and applied these on the central aortic estimates to get pulsatile non-invasive ICP estimates. Thereafter we used the transfer function that gave the best spectral correlation, between the ICP estimates and the ICP signals, on the entire cohort. With this approach we created pulsatile ICP estimates that were completely non-invasive (except the central line necessary for the blood pressure computations). The parameters for the invasively obtained ICP waveform were compared to the parameters for the estimated ICP in 137,512 six second time windows for the 29 individuals. For the first approach the mean absolute difference in invasive versus noninvasive MWA was calculated to be $1.9 \pm 1.0 \mathrm{mmHg}$. For the second approach the mean absolute difference was $1.6 \pm 1.0 \mathrm{mmHg}$ for the 29 individuals.

The conclusion from the study was that the method gave fairly good estimates of the MWA for about a third of the individuals. This is illustrated in Figure 1, where an example of the completely non-invasive approach is shown. Here it becomes evident that a significant amount of the ICP waveform is correctly reproduced. However, the results indicate that the assumption of a linear system linking central aortic blood pressure to ICP is too simplistic, and that a larger part of the complexity of the system must be included for more successful results. As is, this approach is therefore not a reliable method for use in clinical patient management, but it should be investigated further with a larger range of ICP values in order to unequivocally conclude on the usefulness of aortic signals for pulsatile ICP estimation. 

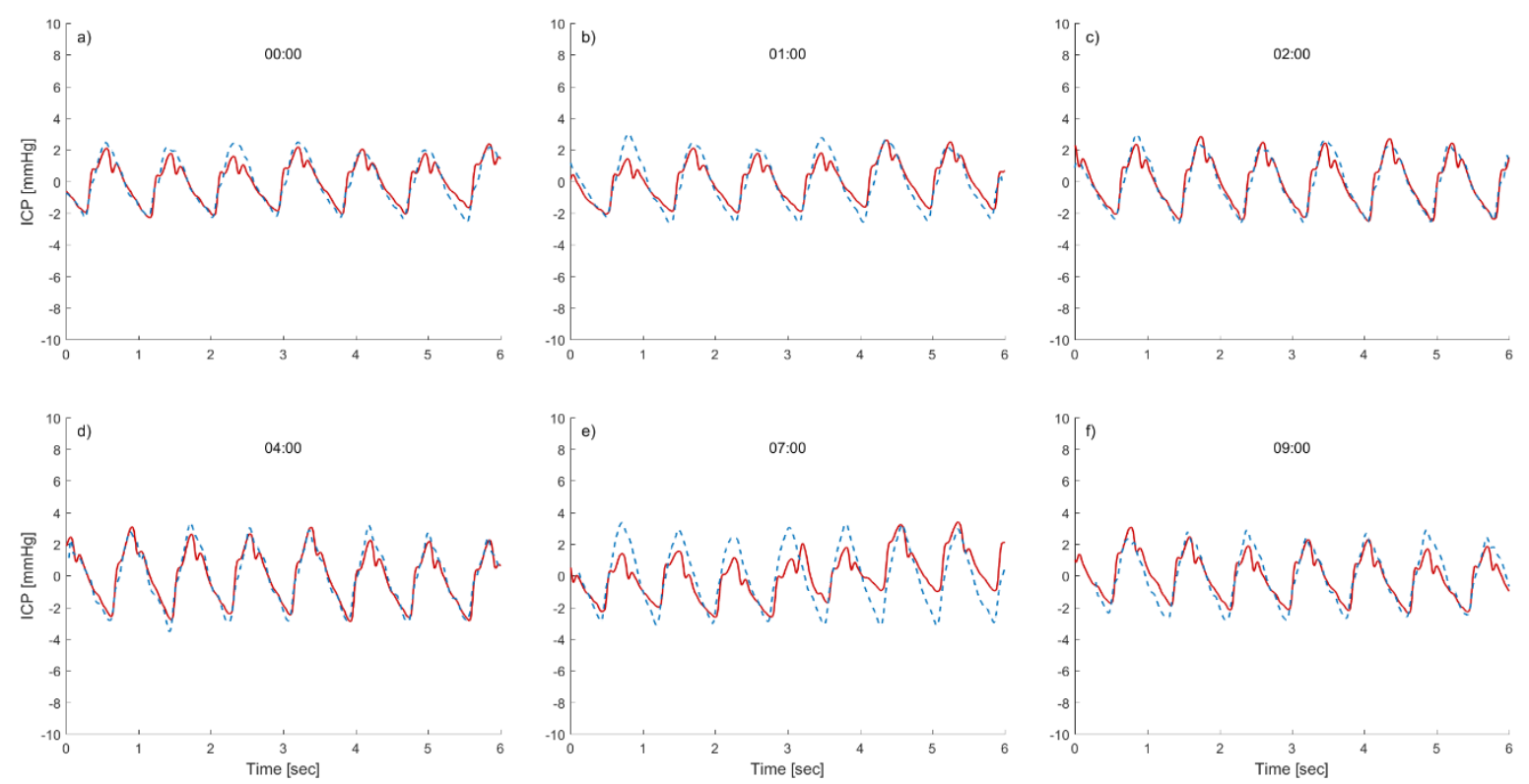

Figure 1. Non-invasively estimated ICP waveforms (interrupted blue lines) superimposed on the invasive ICP waveforms (continuous red lines) for six different time windows of six second duration. The results are shown at midnight, 01:00 am, 02:00 am, 04:00 am, 07:00 am and 09:00 am for the same patient. The Figure is taken from the paper by Evensen et al. ${ }^{7}$ (https://creativecommons.org/licenses/by/4.0/legalcode).

\section{References}

1. Raboel P, Bartek J, Andresen M, Bellander B, Romner B. Intracranial pressure monitoring: invasive versus non-invasive methods - a review. Critical care research and practice. 2012;2012.

2. Harary M, Dolmans RG, Gormley WB. Intracranial Pressure Monitoring-Review and Avenues for Development. Sensors. 2018;18(2):465.

3. Kashif FM, Verghese GC, Novak V, Czosnyka M, Heldt T. Model-based noninvasive estimation of intracranial pressure from cerebral blood flow velocity and arterial pressure. Science translational medicine. 2012;4(129):129ra144-129ra144.

4. Eide P, Czosnyka M, Sorteberg W, Pickard J, Smielewski P. Association between intracranial, arterial pulse pressure amplitudes and cerebral autoregulation in head injury patients. Neurological research. 2007;29(6):578-582.

5. Schmidt B, Schwarze JJ, Czosnyka M, Sander D, Wittich I, Klingelhöfer J. A method for a simulation of continuous intracranial pressure curves. Computers and biomedical research. 1998;31(4):231-243.

6. Schmidt B, Klingelhöfer J. Clinical applications of a non-invasive ICP monitoring method. European journal of ultrasound. 2002;16(1-2):37-45.

7. Evensen KB, O'Rourke M, Prieur F, Holm S, Eide PK. Non-invasive Estimation of the Intracranial Pressure Waveform from the Central Arterial Blood Pressure Waveform in Idiopathic Normal Pressure Hydrocephalus Patients. Scientific reports. 2018;8(1):4714.

8. Kim MO, Eide PK, O'Rourke MF, Adji A, Avolio AP. Intracranial Pressure Waveforms are More Closely Related to Central Aortic than Radial Pressure Waveforms: Implications for Pathophysiology and Therapy. Acta neurochirurgica Supplement. 2016;122:61-64.

9. Avezaat C, Van Eijndhoven J, Wyper D. Cerebrospinal fluid pulse pressure and intracranial volume-pressure relationships. Journal of Neurology, Neurosurgery \& Psychiatry. 1979;42(8):687-700. 
10. Takizawa H, Gabra-Sanders T, Miller DJ. Changes in the cerebrospinal fluid pulse wave spectrum associated with raised intracranial pressure. Neurosurgery. 1987;20(3):355-361.

11. Robertson CS, Narayan RK, Contant CF, et al. Clinical experience with a continuous monitor of intracranial compliance. Journal of neurosurgery. 1989;71(5):673-680.

12. Eide PK. The correlation between pulsatile intracranial pressure and indices of intracranial pressure-volume reserve capacity: results from ventricular infusion testing. Journal of neurosurgery. 2016;125(6):1493-1503.

Karen Brastad Evensen ${ }^{1,2}$

Per Kristian Eide Eis $^{2,3}$

${ }^{1}$ Department of Informatics, University of Oslo, Oslo, Norway;

${ }^{2}$ Department of Neurosurgery, Oslo University Hospital - Rikshospitalet, Oslo, Norway;

${ }^{3}$ Institute of Clinical Medicine, Faculty of Medicine, University of Oslo, Oslo, Norway. 